\title{
ENO3 promoted the progression of NASH by negatively regulating ferroptosis via elevation of GPX4 expression and lipid accumulation
}

\author{
Di Lu" ${ }^{1 \#}$, Qiaoyun Xia ${ }^{1 \#}$, Zhiyu Yang ${ }^{1}$, Shanjun $\mathrm{Gao}^{2}$, Suofeng Sun ${ }^{1}$, Xiaoying Luo ${ }^{1}$, Zhen $\mathrm{Li}^{2}$, \\ Xiulei Zhang ${ }^{2}$, Shuangyin $\mathrm{Han}^{1}$, Xiuling $\mathrm{Li}^{1}$, Mingbo Cao ${ }^{1}$ \\ ${ }^{1}$ Department of Gastroenterology, Henan Provincial People's Hospital, People's Hospital of Zhengzhou University, School of Clinical Medicine, \\ Henan University, Zhengzhou, China; ${ }^{2}$ Microbiome Laboratory, Henan Provincial People's Hospital, People's Hospital of Zhengzhou University, \\ Zhengzhou, China \\ Contributions: (I) Conception and design: M Cao, D Lu; (II) Administrative support: X Li; (III) Provision of study materials or patients: Z Yang; (IV) \\ Collection and assembly of data: Q Xia, D Lu; (V) Data analysis and interpretation: D Lu; (VI) Manuscript writing: All authors; (VII) Final approval \\ of manuscript: All authors. \\ \#These authors contributed equally to this work and are co-first authors. \\ Correspondence to: Xiuling Li; Mingbo Cao. Department of Gastroenterology, Henan Provincial People's Hospital, People's Hospital of Zhengzhou \\ University, School of Clinical Medicine, Henan University, Zhengzhou 450003, China. Email: li_xiuling123@126.com; mingbocao@126.com.
}

Background: ENO3 expression is upregulated in Non-alcoholic fatty liver disease (NAFLD) patient tissues, demonstrated that ENO3 might play crucial roles in NAFLD. However, the mechanism of ENO3 in NAFLD remains unclear. Therefore, this study aimed to investigate the regulatory mechanism of ENO3 in the progression of non-alcoholic steatohepatitis (NASH) in vivo and vitro NASH model.

Methods: In vivo and vitro NASH model were established by methionine-choline deficient (MCD)-diet feeding and high free fatty acid (HFFA) induction in L02 cells. Loss and gain function of ENO3 and GPX4 was performed to study the mechanism in NASH. Western blot was used to detect the expression of ENO3 and GPX4. Hematoxylin and eosin (H\&E), picrosirius Red and Oil Red O staining was used to evaluate histopathology of liver in NASH model. Ferroptosis indicators were measured by assay kits according to the manufacturer's instructions.

Results: NASH mouse model was successfully established induced by MCD diet with steatosis, inflammatory infiltration, ballooning and fibrosis observed in the liver tissue. The expression of ENO3 and GPX4 was significantly elevated while ferroptosis was inhibited in NASH mice and cell model. Upregulation of both ENO3 and GPX4 could promote the lipid accumulation in L02 cells. In addition, overexpressed ENO3 attenuated the status of ferroptosis.

Conclusions: In the present study, we demonstrate that ENO3 promoted the progression of NASH by negatively regulating ferroptosis via elevating GPX4 expression and lipid accumulation. These findings provided solid foundation for the mechanism of ferroptosis on the progression of NASH regulated by ENO3, suggesting that ENO3 may be a potential therapeutic target for NASH.

Keywords: Non-alcoholic steatohepatitis (NASH); ENO3; ferroptosis; lipid accumulation; GPX4

Submitted Dec 30, 2020. Accepted for publication Apr 17, 2021.

doi: $10.21037 / \mathrm{atm}-21-471$

View this article at: http://dx.doi.org/10.21037/atm-21-471 


\section{Introduction}

Non-alcoholic fatty liver disease (NAFLD) refers to a clinicopathological syndrome characterized by excessive accumulation of fat in liver cells that is not caused by alcohol or other clear liver damage factors, and includes non-alcoholic fatty liver (NAFL), non-alcoholic steatohepatitis (NASH), and related cirrhosis (1). NAFLD refers to pathological conditions of accumulation of excess, non-alcoholic fat in the liver. But, NASH refers to the liver inflammation and damage caused by a buildup of fat in the liver. NAFLD has become the most common and significant chronic liver disease in the world due to the increasing prevalence of obesity in adults and children (2). NASH is a more serious form of NAFLD that can progress to liver cirrhosis and liver cancer. Although it has been demonstrated that steatosis, inflammation, and fibrosis are the main pathological processes contributing to the development of NASH (3), the detailed pathogenesis and pathophysiological processes of NASH remain unclear.

Regulated cell death (RCD) is pivotal in directing the severity and outcome of liver injury. Hepatocyte cell death is a critical event in the progression of liver disease due to resultant inflammation leading to fibrosis. Apoptosis, necrosis, necroptosis, autophagy, and recently, pyroptosis and ferroptosis, have all been investigated in the pathogenesis of various liver diseases (4). Lipid peroxidation, ROS accumulation, and increased liver iron stores (both in the parenchymal and non-parenchymal compartment) have been noted in patients with NASH (5). Furthermore, antioxidants such as vitamin $\mathrm{E}$ and iron reduction by phlebotomy have been shown to improve liver chemistries, which is a surrogate for hepatitis, in patients with NAFLD/NASH (6,7). In a recent study, Qi et al. observed an induction of GPX4 protein after 24 weeks of Western diet or 10 days of MCD feeding in mice (8). Therefore, it is worth exploring whether ferroptosis plays an important role in the pathogenesis of NAFLD/NASH.

Ferroptosis is a newly discovered programmed cell death, which is characterized by the accumulation of a large amount of iron ions and lipid peroxidation in the process of cell death (9). Ferroptosis is primarily induced by catalyzing the occurrence of lipid peroxidation of unsaturated fatty acids on the cell membrane under the action of divalent iron or ester oxygenase (10). It is also manifested via the reduction of the core enzyme glutathione peroxidase 4 (GPX4) in the antioxidant system, which exhibits anti-oxidative activity by reducing phospholipid hydroperoxides in membranes (11).
GPX4 is a key regulator of ferroptosis, which can convert lipid hydroperoxides to lipid alcohol to protect cells from death, which can convert lipid hydroperoxides to lipid alcohol to protect cells from death (12). First identified in 1982 as the second mammalian glutathione peroxidase (13), Yang and Friedmann group demonstrated in 2014 that the selenoperoxidase GPX4 is the key upstream regulator of ferroptosis $(14,15)$. The role of GPX4 as the main regulator in the ferroptotic process is based on its unique function to reduce complex hydroperoxides including phospholipid hydroperoxides and cholesterol hydroperoxides to their corresponding counterparts, thereby interrupting the lipid peroxidation chain reaction (16). Numerous studies have shown that this mode of death is closely related to various diseases, including nervous system diseases, tumors, ischemiareperfusion injury diseases, as well as kidney injury and iron metabolism diseases (17). Recently, an increasing number of studies have found that ferroptosis is involved in the pathogenesis of NASH (18). Tsurusaki et al. [2019] demonstrated that hepatic ferroptosis inhibition protected $\mathrm{NASH}$ by suppressing the subsequent infiltration of immune cells and inflammatory reaction (19). Qi et al. [2020] revealed that ferroptosis affected the progression of NASH by modulating lipid peroxidation-mediated cell death in mice (8). These results suggested that novel prevention and treatment methods for NASH are expected to be developed via the regulation of ferroptosis. However, the regulation mechanism of ferroptosis in the pathogenesis of NASH remains unclear.

With the rapid development of advanced technologies, an increasing number of genes or non-coding ribonucleic acids (RNAs) have been found to play key roles in the pathogenesis of NASH, such as cluster of designation 82 (CD82), hepatocyte nuclear factor 4 alpha (HNF4A), klotho beta (KLB), and microRNA-223 (miR-223) (20-23). Enolase 3 (ENO3) encodes the $\beta$-subunit of enolase, which is distributed in various tissues, including liver, lung, skeletal, and heart (24). However, the mechanism of ENO3 in NAFLD remains unclear. It has been demonstrated that ENO3 accelerates hepatic cholesterol ester accumulation caused by mediating cholesterol ester synthesis (24). Furthermore, previous studies have demonstrated that ENO3 expression is upregulated in NAFLD patient tissues by integrating the analyses of multiple microarray studies (25). These findings demonstrated that ENO3 might play a crucial role in NAFLD via the regulation of ferroptosis. 
Table 1 Sequences of primers

\begin{tabular}{lcl}
\hline Target gene & Primer & Primer sequence \\
\hline ENO3 & GTP-ENO3-F: & 5'-GAAGATTCTAGAGCTAGCGAATTCATGGCCATGCAGAAAATCTT1-3' \\
- OE & GTP-ENO3-R: & 5'-CGCAGATCCTTGCGGCCGCGCGGCCGCTCACTTGGCCTTCGGGTTA-3' \\
GPX4 & GTP-GPX4-F: & 5'-GAAGATTCTAGAGCTAGCGAATTCATGAGCCTCGGCCGCCTTTGCC-3' \\
-OE & GTP-GPX4-R: & 5'-CGCAGATCCTTGCGGCCGCGCGGCCGCTCATGAGTGCCGGTGGAAGGC-3' \\
sh- & ENO3-sh-F: & 5'-CCGGGGAAGTACGATCTTGACTTCACGAATGAAGTCAAGATCGTACTTCC TTITTG-3' \\
ENO3 & ENO3-sh-R: & 5'-AATTCAAAAAAGGAAGTACGATCTTGACTTCATTCGTGAAGTCAAGATCGTACTTCC-3' \\
sh- & GPX4-sh-F: & 5'-CCGGGGACACCGTCTCTCCACAGTTCGAAAACTGTGGAGAGACGGTGTCC TTTTTTG-3' \\
GPX4 & GPX4-sh-R: & 5'-AATTCAAAAAAGGACACCGTCTCTCCACAGTTTTCGAACTGTGGAGAGACGGTGTCC-3' \\
\hline
\end{tabular}

OE, overexpression; sh, shRNA.

Therefore, in the present study, we aimed to investigate the regulatory mechanism of ENO3 in NASH via the regulation of ferroptosis using in vivo and in vitro NASH models. We present the following article in accordance with the ARRIVE reporting checklist (available at http:// dx.doi.org/10.21037/atm-21-471).

\section{Methods}

\section{Cell lines and cell culture}

Normal human liver L02 cell line was purchased from the American Type Culture Collection (ATCC, Bethesda, MD, USA). Cells were cultured in 90\% Dulbecco's modified Eagle's medium (DMEM, 12430054, Gibco, USA) supplemented with $10 \%$ fetal bovine serum (FBS, Gibco, USA), $100 \mu \mathrm{g} / \mathrm{mL}$ penicillin, and $100 \mu \mathrm{g} / \mathrm{mL}$ streptomycin at $37{ }^{\circ} \mathrm{C}$ in a $5 \%$ carbon dioxide $\left(\mathrm{CO}_{2}\right)$-contained incubator under $95 \%$ saturation humidity. L02 cells were treated with high free fatty acid (HFFA, 01383-1G, and P9767-5G, Sigma USA) for 72 hours to establish the NASH cell model.

\section{Cell transfection}

Cell transfection was performed by ENO3 and GPX4 lentiviral transfection harboring with overexpressed (OE) or short hairpin RNA (shRNA) plasmid of ENO3 and GPX4 as previously described (26). Briefly, L02 cells at a density of $3 \times 10^{5}$ cells/well were seeded in a six-well plate prior to transfection. Lentiviral-harbor overexpressed or shRNA targeting ENO3 and GPX4 were transiently transfected into L02 cells when the cell confluence was $60-70 \%$, respectively. The negative control for overexpression (OE-
NC) and negative control for short hairpin RNA (Sh-NC) lentiviral transfection were used as controls. The lentivirus of ENO3 and GPX4 were purchased from Zhengzhou DingHan Biotechnology Co., Ltd. China, and the overexpression and shRNA sequences were as Table 1.

For ferroptosis activation, $0.5 \mu \mathrm{M}$ ferroptosis activator (1S, 3R)-RSL3 (RSL-3) (1219810-16-8, Macklin) was used to treat L02 cells for 24 hours. Transfection efficiency was detected by quantitative reverse transcription-polymerase chain reaction (qRT-PCR).

\section{Western blotting}

Total protein from liver tissue and L02 cells was extracted using Radio-Immunoprecipitation Assay (RIPA) Lysis buffer (P0013, Beyotime, Shanghai, China) and then quantified using a bicinchoninic acid (BCA) protein assay kit (70-PQ0012, MultiSciences, China) according to the manufacturer's instructions. In each sample, $30 \mu \mathrm{g}$ proteins with $20 \%$ protein loading buffer was separated in $10-12 \%$ dodecyl sulfate, sodium salt (SDS)-Polyacrylamide gel electrophoresis (SDS-PAGE) and then transferred onto polyvinylidene fluoride (PVDF) membranes. Next, the membranes were blocked using $5 \%$ lipid-free milk/ Tris Buffered saline Tween (TBST) for 2 hours at room temperature, and subsequently incubated with anti-ENO3 (55234-1-AP, 1:200, proteintech), anti-GPX4 (PAB40411, 1:2,000, Abcam, Bioswamp $\left.{ }^{\mathrm{TM}}\right)$, and anti-glyceraldehyde3-phosphate dehydrogenase (GAPDH) (sc-365062, 1:5,000, Santa Cruz) primary antibodies for 2 hours at $4{ }^{\circ} \mathrm{C}$ overnight, respectively. After incubation with secondary anti-mouse immunoglobulin- G (IgG) antibody (ab205719, 
1:20,000, Abcam, Cambridge, UK) for 1-2 hours at room temperature, the immuno-complexes were finally detected by efficient chemiluminescence (ECL) and the relative expression of OEN3 and GPX4 was analyzed using Image J software (USA).

\section{MCD-diet induced the NASH mouse model}

Eight-week-old C57BL/6 mice, body weight about 22-24 g, male $(n=24)$ were purchased from Beijing Vital River Laboratory Animal Technology Co. Ltd China, were maintained in a pathogen-free environment with access to sterile water and a standard controlled diet. The mice were randomly divided into four groups and were maintained on a MCD diet (Medicience, Yangzhou, China) for 4, 8, and 12 weeks to induce NASH. Liver tissue and blood samples (from the eyeballs of the mice) were harvested for further analyses. Mice on a normal diet were used as the control. All animal experiments were approved by Ethics Committee of Zhengzhou University and were performed in accordance with the requirements outlined by the Animal Care and Ethics Committees of Henan Provincial People's Hospital. Serum alanine aminotransferase (ALT) and aspartate aminotransferase (AST) (obtained from the blood of mice eyeballs) were measured by the automatic biochemical analyzer Chemray 240 and 800 (Shenzhen China), respectively. Triglycerides (TGs) were measured using a triglycerides assay kit (E1025, APPLYGEN, Beijing) according to the manufacturer's instructions.

\section{Hematoxylin and eosin (HE), picrosirius red, and oil red o staining}

Liver tissues were sliced into $8 \mu \mathrm{m}$-thick sections and fixed with $4 \%$ paraformaldehyde at room temperature. For HE staining, the deparaffinized sections were stained in hematoxylin and eosin staining solutions for 5 minutes, respectively. The slides were then dehydrated and sealed with neutral gum. For evaluation of liver fibrosis, picrosirius red staining. For oil red o staining, the sections or L02 cell lines were rinsed with phosphate buffer saline (PBS) and subsequently stained with hematoxylin solution. Next, the sections were stained with oil red o staining solution after incubation in $60 \%$ isopropanol for $10 \mathrm{~min}$. Finally, the sections were washed with running water and were observed under a microscope (Olympus, Japan). We evaluate the hepatic steatosis, inflammation, ballooning and liver fibrosis respectively using microscope of OLYMPUS BX53 according to the NAFLD activity score (NAS) system (27).

\section{Ferroptosis indicators measurement}

Malondialdehyde (MDA) and glutathione transferase (GST) in liver tissue and L02 cells were measured using a MDA assay kit (A003-1, TBA method, Nanjing Jiancheng Bioengineering Institute) and reduced glutathione (GSH) assay kit (A006-1, Nanjing Jiancheng Bioengineering Institute), respectively, according to the manufacturer's instructions. Ferrous ion $(2+)\left(\mathrm{Fe}^{2+}\right)$ concentration was measured using an iron colorimetric assay kit (E1042, APPLYGEN, Beijing) according to the manufacturer's instructions.

\section{Statistical analysis}

All statistical analyses were performed using SPSS 21.0 software (IBM, Armonk, NY, USA). Data were shown as the mean \pm standard deviation of three independent experiments. The student's $t$-test was used to examine the significance between two groups, one way ANOVA was used to examine the significance more than two groups, and $\mathrm{P}<0.05$ was considered statistically significant.

\section{Results}

\section{The MCD diet-induced NASH mouse model was successfully constructed}

In order to explore the role of $\mathrm{ENO} 3$ in NASH, a MCD diet-induced NASH mouse model was established. HE, picrosirius red, and oil red o staining were used to evaluate the pathological features of liver tissue in the NASH model. HE staining showed steatosis inflammatory infiltration, and ballooning in the liver tissue of the mice at 4,8 , and 12 weeks after MCD feeding (Figure 1A). Picrosirius red staining revealed that the fibrosis level of the liver tissue was dramatically elevated in the MCD-diet-induced NASH mouse model at 4, 8, and 12 weeks after MCD feeding (Figure 1B). Oil red o staining also confirmed steatosis in MCD-diet-induced NASH mouse model (Figure 1C). Serum liver injury markers, AST, ALT, and TG, were also measured in the NASH mouse model. As shown in (Figure 1D), the levels of AST and ALT were markedly increased at 4, 8, and 12 weeks after MCD feeding, while 

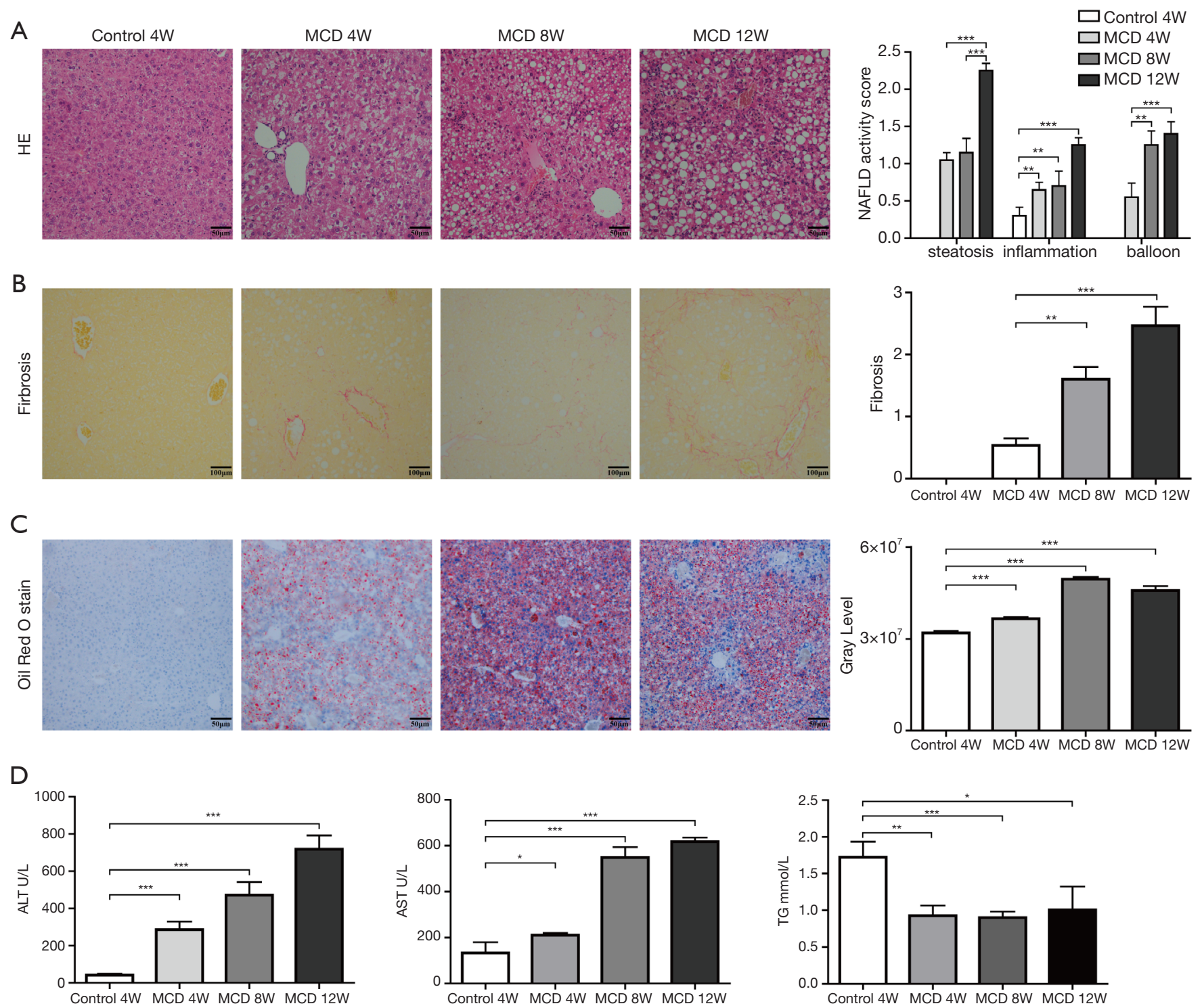

Figure 1 Pathological features of non-alcoholic steatohepatitis in the MCD diet model. (A) HE staining was used to evaluate the lipid accumulation, inflammatory responses, and ballooning in the liver tissue of the MCD diet model mice at 4, 8, and 12 weeks after feeding; (B) Sirius red staining was used to evaluate fibrosis in the liver tissue of the MCD diet model mice at 4, 8, and 12 weeks after MCD diet feeding and statistics with NAFLD activity score system (17); (C) lipid accumulation was observed in the liver tissue of the CDE diet mice model at 4, 8 , and 12 weeks after CDE diet feeding by oil red o staining. Six mice were used for analyses at each time point; (D) measurement of serum liver injury markers ALT, AST, and TG at 4, 8, and 12 weeks after the CDE feeding. The data are shown as the mean \pm standard deviation $(\mathrm{n}=6) ;{ }^{*}, \mathrm{P}<0.05 ;{ }^{* *}, \mathrm{P}<0.01 ;{ }^{* * *}, \mathrm{P}<0.001$, MCD group vs. Control group.

the level of TG was dramatically decreased compared to the control group. These results demonstrated that the MCD diet-induced NASH mouse model was successfully established, and the degree of NASH in the livers of the mice gradually increased with the increased MCD feeding duration.

\section{Changes of ENO3, GPX4, and ferroptosis-related indicators in NASH mouse and cell models}

In order to identify the correlation between ENO3 and $\mathrm{NASH}$, the expression of $\mathrm{ENO} 3$ was detected in the livers of the NASH mice. The results showed that the expression 
of ENO3 was significantly increased in the NASH mice model group with MCD feeding for 4, 8, and 12 weeks compared to the control group (Figure $2 A$ ). Previous studies have demonstrated that GPX4 is downregulated during ferroptosis. Therefore, the expression of GPX4 was measured in the livers of the NASH mice. Results showed that the expression of GPX4 was also significantly increased in the NASH mice model group with MCD feeding for 4, 8 , and 12 weeks compared to the control group (Figure $2 A$ ), which is consistent with the expression changes of ENO3 in the NASH mice model. These results demonstrated ENO3 might participate in NASH-related ferroptosis. Further analysis showed that MDA was significantly decreased, while the concentration of GSH was dramatically increased in the NASH mice model group with MCD feeding for 4, 8 , and 12 weeks. We also found that the $\mathrm{Fe}^{2+}$ concentration was significantly decreased in the NASH mice model group with MCD feeding for 4, 8, and 12 weeks (Figure 2B).

In order to further confirm these results, a NASH cell model was constructed using the L02 cell line by treating with HFFA for 72 hours. Oil red o staining revealed steatosis in these $\mathrm{L} 02$ cells (Figure 2C). The results also showed that the concentration of MDA was significantly decreased, while the concentration of GSH was increased in L02 cells after treatment with HFFA (Figure 2C). In addition, the expression of both ENO3 and GPX4 were significantly increased in L02 cells after HFFA treatment (Figure 2D). These results were consistent with the results in the mouse model, demonstrating that the inhibition of ferroptosis might be affected by the elevated expression of ENO3 and GPX4.

\section{ENO3 affected the lipid deposition and ferroptosis indicators in L02 cells}

In order to investigate whether ENO3 could affect ferroptosis, the loss and gain functions of $\mathrm{ENO} 3$ in $\mathrm{L} 02$ cells were performed. As shown in Figure 3A, ENO3 was significantly overexpressed or suppressed in the $\mathrm{ENO} 3 \mathrm{OE}$ and ENO3 knock-down (KD) groups compared to the wild type (WT) group. The expression of GPX4 exhibited the same trend in the expression level of ENO3 in the ENO3 $\mathrm{OE}$ and ENO3 KD groups. However, it showed that the expression of ENO3 was significantly elevated, while the expression of GPX4 was downregulated in the ENO3 group after treatment with the ferroptosis activator RSL3 (Figure $3 A$ ). Further analysis showed that lipid deposition was significantly aggravated in both the ENO3 OE and ENO3 OE-RSL3 groups, and was inhibited in the ENO3 KD group compared to the WT group. Also, lipid deposition was mitigated in the ENO3 OE-RSL3 group relative to the ENO3 group (Figure $3 B$ ). In addition, the concentration of MDA was significantly decreased in the $\mathrm{ENO} 3 \mathrm{OE}$ group, and was elevated in the $\mathrm{ENO} 3 \mathrm{KD}$ and ENO3 OE-RSL3 groups compared to the WT group (Figure 3C). Meanwhile, the changes in GSH exhibited opposite results to those observed in MDA (Figure 3C).

\section{GPX4 affected lipid deposition in L02 cells}

In order to determine whether ENO3 affected lipid deposition caused by changes in the expression of GPX4, the loss and gain functions of GPX4 in L02 cells were performed. As shown in Figure 4A, the expression of GPX4 was significantly overexpressed in the GPX4 OE group, and was suppressed in the GPX4 KD and GPX4 OERSL3 groups relative to the WT group. Further analysis showed that the overexpression of GPX4 could significantly aggravate lipid deposition, while suppressed GPX4 blocked lipid deposition, compared to the WT group (Figure 4B). However, lipid deposition was significantly attenuated in the GPX4 OE group after treatment with RSL-3 in L02 cells (Figure 4B), suggesting that GPX4 could regulate lipid deposition.

\section{Discussion}

NAFLD is a metabolic stress liver injury closely related to obesity, insulin resistance, and genetic susceptibility, and is considered to be the manifestation of metabolic syndrome in the liver $(28,29)$. NASH is a more serious pathological process in NAFLD that is affected by a variety of concurrent factors, including lipotoxicity, abnormal lipid metabolism, oxidative stress, genetic susceptibility, mitochondrial dysfunction, abnormal production of cytokines and adipocytokines, intestinal microecological disorders, and endoplasmic reticulum stress $(30,31)$. Although the pathogenesis is complicated, it is clear that occurrence and development of NASH is caused by the imbalance of lipid metabolism in liver cells (32). However, the regulatory mechanism of lipid metabolism remains unclear in the progression of NASH. Therefore, it is particularly important to conduct in-depth research on lipid metabolism in the pathogenesis of NASH, in order to prevent the formation and development of NASH. In the present study, we successfully established a MCD diet- 


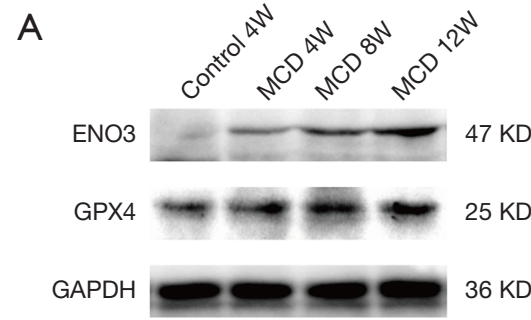

B

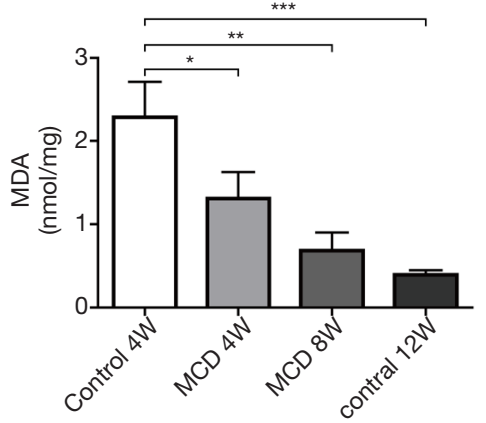

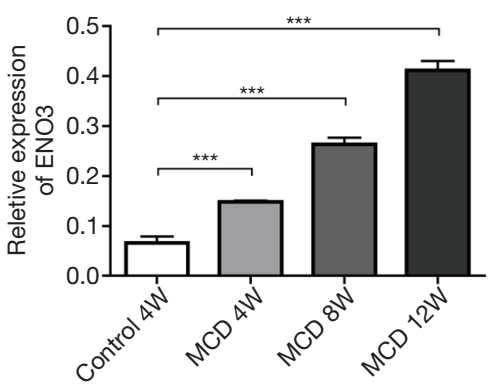

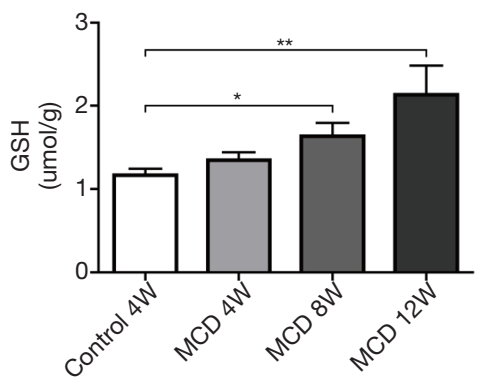

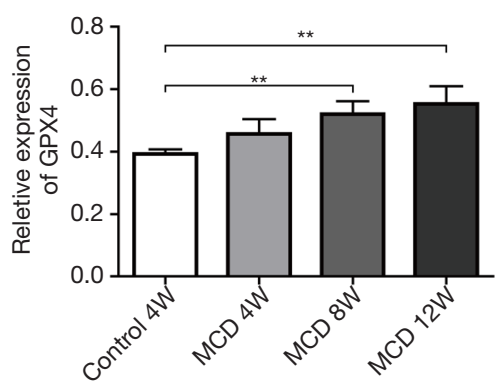

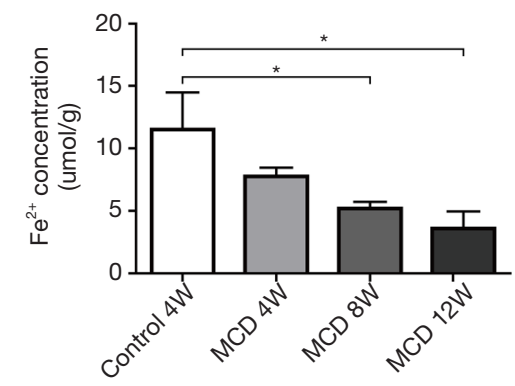

C

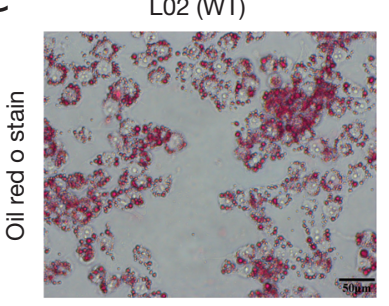

L02 (HFFA72h)
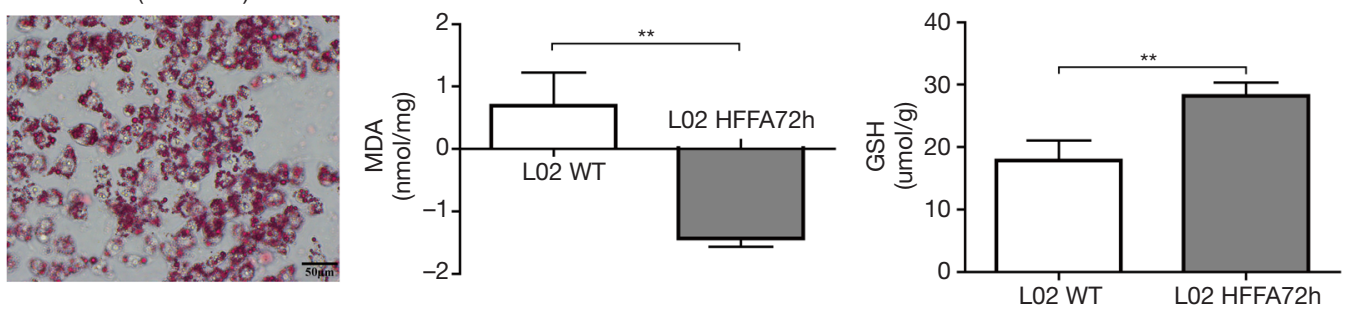

D
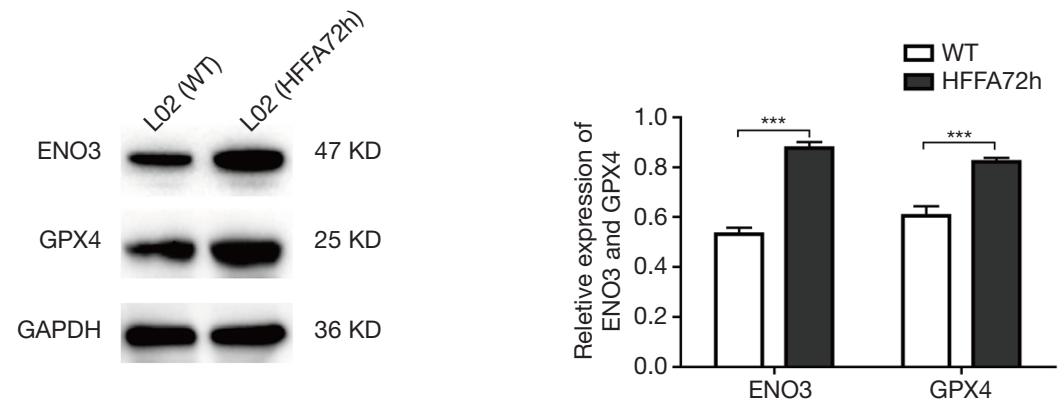

Figure 2 Changes of ENO3, GPX4, and ferroptosis-related indicators in the NASH mice and cell models. (A) The protein expression of ENO3 and GPX4 in the liver tissue of the CDE diet model mice at 4, 8, and 12 weeks after CDE diet feeding was detected by western blotting and qRT-PCR, respectively; GAPDH was used as a control; (B) changes in MDA, GSH, and $\mathrm{Fe}^{2+}$ concentration in the liver tissue of the CDE diet model mice at 4, 8, and 12 weeks after CDE diet feeding; (C) oil red o staining was used to detect lipid accumulation and the levels of MDA and GSH in L02 cell lines after HFFA treatment for 72 hours; (D) the protein expression of ENO3 and GPX4 in the L02 cell line after HFFA treatment for 72 hours was detected by western blotting, and the relative expression of ENO3 and GPX4 was calculated by Image J. GAPDH was used as a control. Each set of samples was repeated three times. The data are shown as the mean \pm standard deviation $(\mathrm{n}=6) ;{ }^{*}, \mathrm{P}<0.05 ;{ }^{* *}, \mathrm{P}<0.01 ;{ }^{* * *}, \mathrm{P}<0.001, \mathrm{MCD}$ group vs. Control group or HFFA $72 \mathrm{~h}$ group vs. WT group. 
A
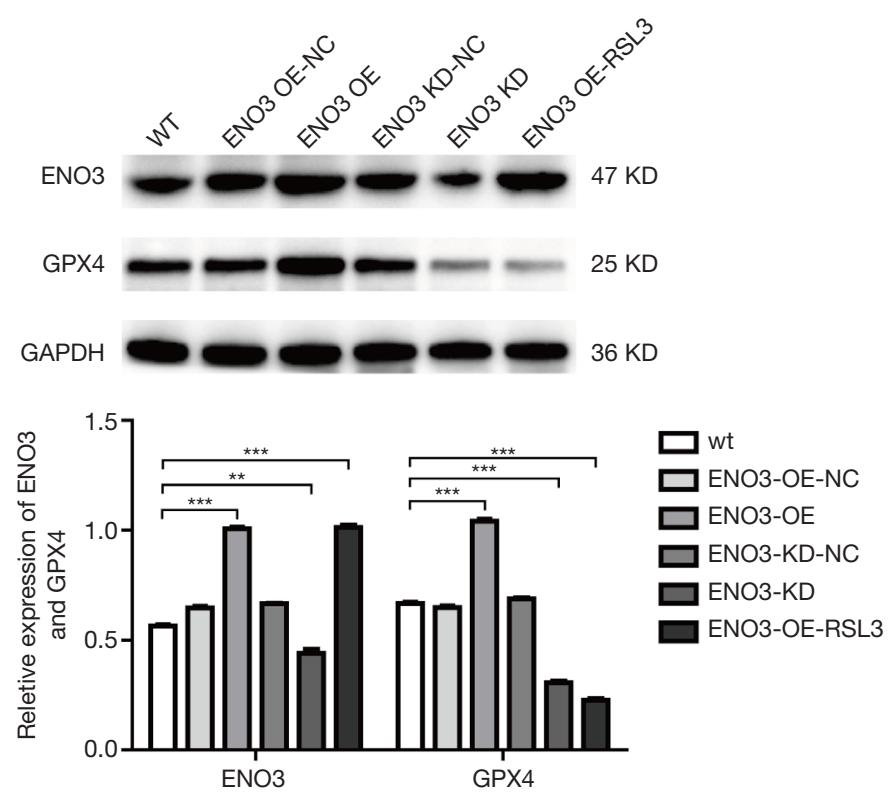

B
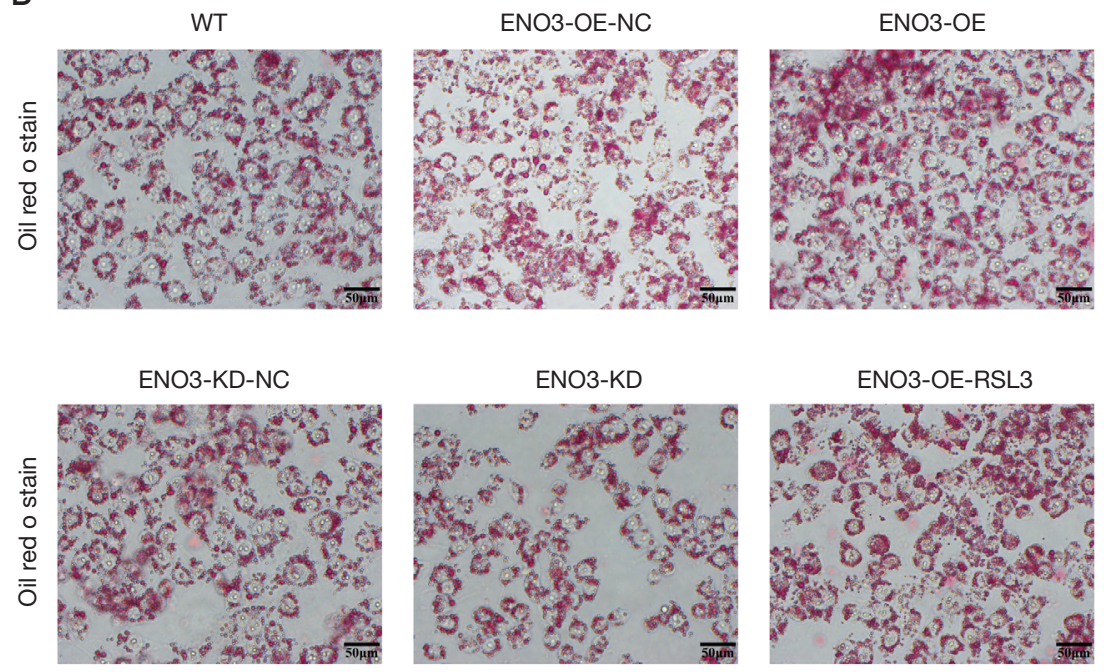

C
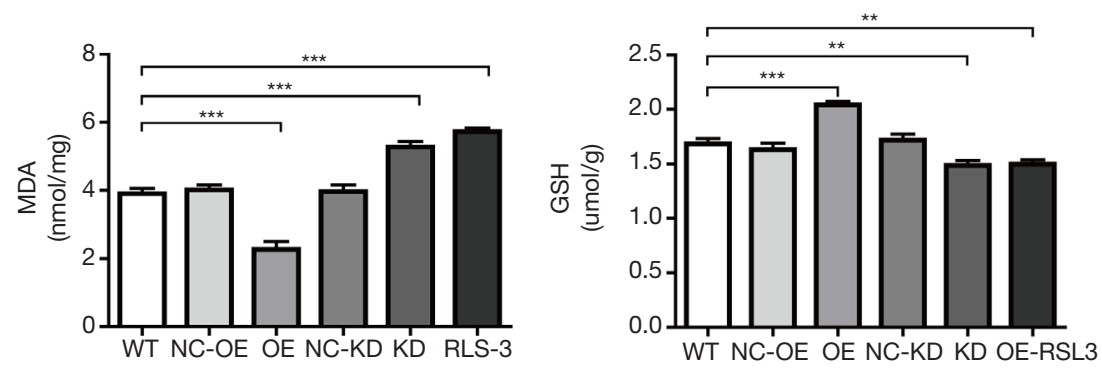

Figure 3 ENO3 affected the lipid deposition and ferroptosis indicators in L02 cells. (A) The expression of ENO3 and GPX4 was detected by western blotting in the overexpressed/suppressed ENO3 L02 cells and in the overexpressed ENO3 L02 cells treated with RSL3. GAPDH was used as a control; (B) Oil red o staining was used to detect the lipid accumulation in the different groups; (C) the concentrations of MDA and GSH changed in the L02 cell lines in the different groups. Each set of samples was repeated three times. The data are shown as the mean \pm standard deviation $(\mathrm{n}=6) ;{ }^{* *} \mathrm{P}<0.01 ;{ }^{* * *} \mathrm{P}<0.001$, vs. WT group. 
A
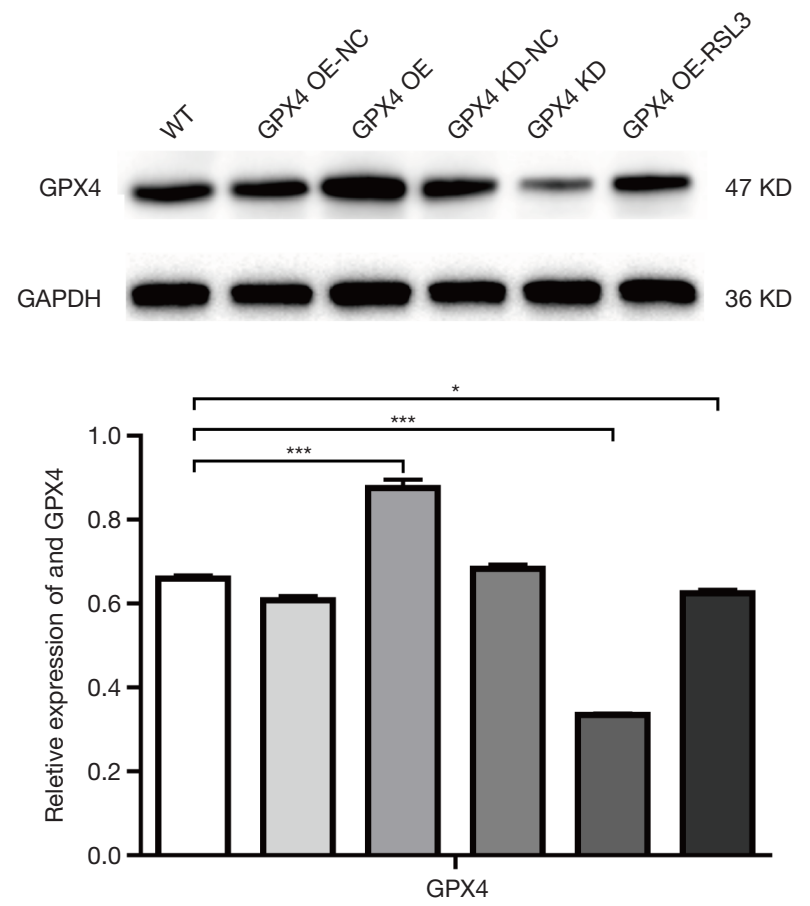

WT
GPX4-OE-NC
GPX4-OE
GPX4-KD-NC
GPX4-KD
GPX4-OE-RSL3

B

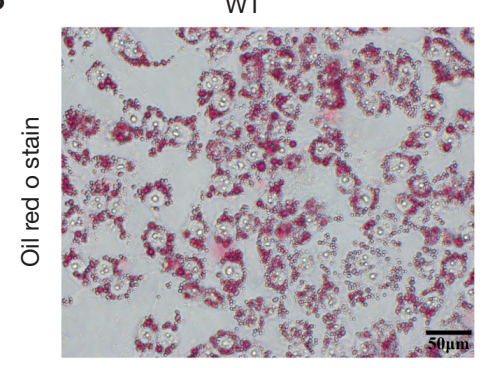

GPX4 KD-NC

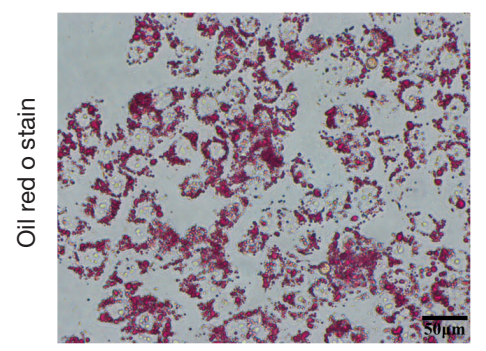

GPX4 OE-NC

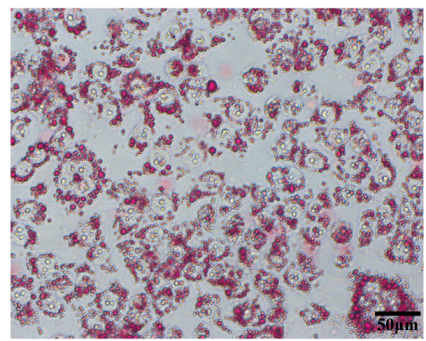

GPX4 KD

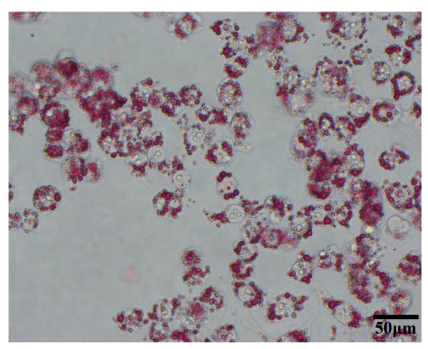

GPX4 OE

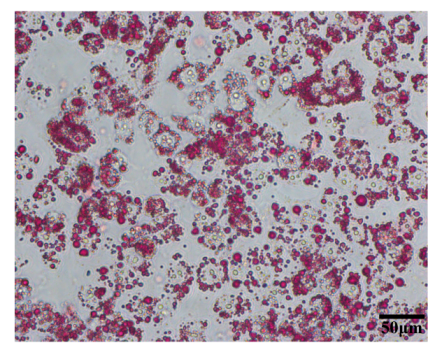

GPX4 OE-RSL3

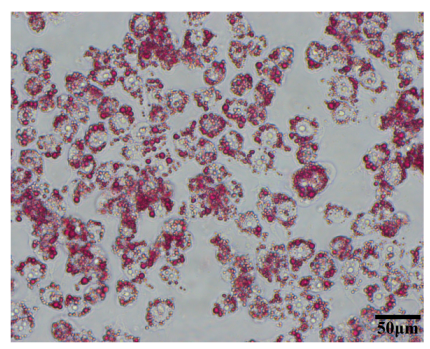

Figure 4 GPX4 affected the lipid deposition in L02 cells. (A) The expression of GPX4 was detected by western blotting in overexpressed/ suppressed GPX4 L02 cells and in the overexpressed GPX4 L02 cells treated with RSL3. GAPDH was used as a control; (B) Oil red o staining was used to detect the lipid accumulation in different groups. Each set of samples was repeated three times. The data are shown as the mean \pm standard deviation $(\mathrm{n}=6) ;{ }^{*}, \mathrm{P}<0.05 ;{ }^{* * *}, \mathrm{P}<0.001$, vs. WT group. 
induced NASH model with inflammatory infiltration, ballooning, steatosis, and fibrosis in the livers of mice, and investigated the regulatory mechanism of lipid accumulation in NASH.

An increasing numbers of studies have shown that various kinds of regulatory factors are involved in different processes in the development of NASH. For example, microRNA-223 ameliorates NASH and cancer by affecting inflammation in hepatocytes, and cellular communication network factor 1 (CCN1) also promotes hepatic steatosis and inflammation in NASH (20,33). Membrane-boundacyltransferase 7 (MBOAT7) suppresses the progression of NAFLD by driving acylation of lysophosphatidylinositol (LPI) lipids (34). Recently, it was demonstrated that ENO3 expression is upregulated in the tissues of NAFLD patients by integrating analyses of multiple microarray studies (24). Elam et al. also showed that ENO3 expression was significantly higher in the livers of morbidly obese women compared with women who had experienced massive weight loss (35). Consistent with these results, our study showed that the expression of ENO3 was significantly increased in the MCD diet-induced NASH mouse model and LFFAinduced NASH in vitro model, demonstrating that ENO3 might play a critical role in the progression of NASH. ENO3 encodes the $\beta$-subunit of enolase expressed in various tissues, including liver, lung, skeletal, and heart (24). It has been demonstrated that ENO3 could accelerate hepatic cholesterol ester accumulation by mediating cholesterol ester synthesis via increasing lipid delivery to liver (24). In the present study, we showed that the overexpression of ENO3 could promote the lipid accumulation in L02 cells. However, the mechanism through which ENO3 affects the lipid accumulation in the progression of NASH remains unclear.

Ferroptosis is a newly discovered form of programmed death, which is different from other death procedures such as apoptosis, necrosis, and autophagy (17). The mechanism of iron death may be mainly related to lipid and lipid peroxide accumulation, amino acid antioxidant system imbalance, iron ion metabolism disorder, etc. Ferroptosis is closely related to the occurrence of many diseases, and several studies have shown that ferroptosis could participate in NASH. Tsurusaki et al. [2019] demonstrated that ferroptosis inhibition almost completely protected hepatocytes from necrotic death and suppressed the subsequent infiltration of immune cells and inflammatory reaction in NASH (19). Recently, Wei et al. [2020] and Qi et al. [2020] revealed that ferroptosis promoted the progression of NASH by mediating Mitofusin 2 (Mfn2) and inositol-requiring enzyme 1 alpha (IRE $\alpha$ ) (36), as well as lipid peroxidation (8), respectively. In the present study, we found that that ferroptosis was gradually suppressed as NASH worsened, even in the presence of liver fibrosis, which is inconsistent with the results of previous studies. These findings showed that in order to prevent the progression of $\mathrm{NASH}$, ferroptosis might serve as a repair or resistance regulator to mitigate liver injury in the early stage of NASH. It has also been demonstrated that mevalonate, transsulfide, and heat shock protein signaling played important roles in ferroptosis (9,37-39). However, the function of ENO3 in ferroptosis was rarely investigated. In the present study, we showed that the overexpression of ENO3 could inhibit ferroptosis in the progression of NASH. Further analysis showed that ferroptosis induced by RSL-3 could attenuate the lipid accumulation in L02 cells, demonstrating that ferroptosis regulated the lipid accumulation in the progression of NASH. The processes of lipid synthesis, storage and degradation are finely regulated and associated with ferroptosis (40). Inhibition of stearoyl CoA desaturase 1 (SCD1), an enzyme that catalyzes the rate-limiting step in monounsaturated fattyacid synthesis, can induce both ferroptosis and apoptosis by decreasing CoQ10 and unsaturated fatty acyl chains in membrane phospholipids, and increasing long-chain saturated ceramides (41). While the inhibition of $\beta$-oxidation can restore the sensitivity of tumor cells to ferroptosis (42). Moreover, lipophagy, the degradation of intracellular lipid droplets (LDs) via autophagy, promotes ferroptosis induced by RSL3 in hepatocytes by decreasing lipid storage, and subsequent lipid peroxidation (43). Taken together, ENO3 promoted the progression of NASH by elevating lipid accumulation via negative regulation of ferroptosis.

Ferroptosis is also characterized by the loss of GPX4 activity (44). GPX4 is one of the most important seleniumdependent GPXs. It is characterized by iron-dependent production of reactive oxygen species (ROS) and the consequent non-apoptotic form of cell death $(12,45,46)$. In the present study, we showed that GPX4 was significantly elevated in the MCD diet-induced NASH mice model and LFFA induced NASH in vitro model, which is consistent with the changes in ENO3 expression. We also found that the overexpression of GPX4 could promote lipid accumulation in L02 cells, which decreased with RSL-3 treatment, suggesting that GPX4 could contribute to lipid accumulation in the progression of NASH. In addition, we showed that the expression of GPX4 was elevated 
with $\mathrm{ENO} 3$ overexpression, suggesting that $\mathrm{ENO} 3$ might regulate ferroptosis by regulating the expression of GPX4. However, the regulatory mechanism of ENO3 to GPX4 in the progression of NASH needs to be further studied.

\section{Conclusions}

In summary, we demonstrated that upregulation of ENO3 promoted the progression of NASH by negatively regulating ferroptosis via elevation of GPX4 expression and lipid accumulation, suggesting that ENO3 may be a therapeutic target for NASH.

\section{Acknowledgments}

Funding: This work was supported by the Natural Science Foundation of Henan Province (No. 202300410394), the Henan Provincial People's Hospital, the Science and Technology Department of Henan Province (CN) (Grant 192102310049), and the National Natural Science Foundation of China (No. 81772670).

\section{Footnote}

Reporting Checklist: The authors have completed the ARRIVE reporting checklist. Available at http://dx.doi. org/10.21037/atm-21-471

Data Sharing Statement: Available at http://dx.doi. org/10.21037/atm-21-471

Conflicts of Interest: All authors have completed the ICMJE uniform disclosure form (available at http://dx.doi. org/10.21037/atm-21-471). The authors have no conflicts of interest to declare.

Ethical Statement: The authors are accountable for all aspects of the work in ensuring that questions related to the accuracy or integrity of any part of the work are appropriately investigated and resolved. All animal experiments were approved by Ethics Committee of Zhengzhou University and were performed in accordance with the requirements outlined by the Animal Care and Ethics Committees of Henan Provincial People's Hospital.

Open Access Statement: This is an Open Access article distributed in accordance with the Creative Commons Attribution-NonCommercial-NoDerivs 4.0 International
License (CC BY-NC-ND 4.0), which permits the noncommercial replication and distribution of the article with the strict proviso that no changes or edits are made and the original work is properly cited (including links to both the formal publication through the relevant DOI and the license). See: https://creativecommons.org/licenses/by-nc-nd/4.0/.

\section{References}

1. Yagai T, Miyajima A, Tanaka M. Semaphorin 3E secreted by damaged hepatocytes regulates the sinusoidal regeneration and liver fibrosis during liver regeneration. Am J Pathol 2014;184:2250-9.

2. Anty R, Gual P. Pathogenesis of non-alcoholic fatty liver disease. Presse Med 2019;48:1468-83.

3. Ferro D, Baratta F, Pastori D, et al. New Insights into the Pathogenesis of Non-Alcoholic Fatty Liver Disease: Gut-Derived Lipopolysaccharides and Oxidative Stress. Nutrients 2020;12:2762.

4. Shojaie L, Iorga A, Dara L. Cell Death in Liver Diseases: A Review. Int J Mol Sci 2020;21:9682.

5. Nelson JE, Wilson L, Brunt EM, et al. Relationship between the pattern of hepatic iron deposition and histological severity in nonalcoholic fatty liver disease. Hepatology 2011;53:448-57.

6. Sanyal AJ, Chalasani N, Kowdley KV, et al. Pioglitazone, vitamin $\mathrm{E}$, or placebo for nonalcoholic steatohepatitis. $\mathrm{N}$ Engl J Med 2010;362:1675-85.

7. Valenti L, Moscatiello S, Vanni E, et al. Venesection for non-alcoholic fatty liver disease unresponsive to lifestyle counselling--a propensity score-adjusted observational study. QJM 2011;104:141-9.

8. Qi J, Kim JW, Zhou Z, et al. Ferroptosis Affects the Progression of Nonalcoholic Steatohepatitis via the Modulation of Lipid Peroxidation-Mediated Cell Death in Mice. Am J Pathol 2020;190:68-81.

9. Chen DF, Sun WJ, Liu KJ, et al. Current epidemiology and pathogenesis of non-alcoholic fatty liver diseaseassociated liver cancer. Zhonghua Gan Zang Bing Za Zhi 2017;25:111-4.

10. Magusto J, Majdi A, Gautheron J. Cell death mechanisms in non-alcoholic steatohepatitis. Biol Aujourdhui 2020;214:1-13.

11. Chen J, Yang X, Fang X, et al. The role of ferroptosis in chronic diseases. Zhejiang Da Xue Xue Bao Yi Xue Ban 2020;49:44-57.

12. Forcina GC, Dixon SJ. GPX4 at the Crossroads of Lipid Homeostasis and Ferroptosis. Proteomics 
2019; 19:e1800311.

13. Ursini F, Maiorino M, Valente M, et al. Purification from pig liver of a protein which protects liposomes and biomembranes from peroxidative degradation and exhibits glutathione peroxidase activity on phosphatidylcholine hydroperoxides. Biochim Biophys Acta 1982;710:197-211.

14. Yang WS, SriRamaratnam R, Welsch ME, et al. Regulation of ferroptotic cancer cell death by GPX4. Cell 2014;156:317-31.

15. Friedmann Angeli JP, Schneider M, Proneth B, et al. Inactivation of the ferroptosis regulator $\mathrm{Gpx} 4$ triggers acute renal failure in mice. Nat Cell Biol 2014;16:1180-91.

16. Seibt TM, Proneth B, Conrad M. Role of GPX4 in ferroptosis and its pharmacological implication. Free Radic Biol Med 2019;133:144-52.

17. Zhang X, Huang Z, Xie Z, et al. Homocysteine induces oxidative stress and ferroptosis of nucleus pulposus via enhancing methylation of GPX4. Free Radic Biol Med 2020;160:552-65.

18. Le Y, Zhang Z, Wang C, et al. Ferroptotic Cell Death: New Regulatory Mechanisms for Metabolic Diseases. Endocr Metab Immune Disord Drug Targets 2020. [Epub ahead of print]. doi: 10.2174/1871530320666200 731175328.

19. Tsurusaki S, Tsuchiya Y, Koumura T, et al. Hepatic ferroptosis plays an important role as the trigger for initiating inflammation in nonalcoholic steatohepatitis. Cell Death Dis 2019;10:449.

20. He Y, Hwang S, Cai Y, et al. MicroRNA-223 Ameliorates Nonalcoholic Steatohepatitis and Cancer by Targeting Multiple Inflammatory and Oncogenic Genes in Hepatocytes. Hepatology 2019;70:1150-67.

21. Ji F, Liu Y, Hao JG, et al. KLB gene polymorphism is associated with obesity and non-alcoholic fatty liver disease in the Han Chinese. Aging (Albany NY) 2019;11:7847-58.

22. Habibzadeh P, Honarvar B, Silawi M, et al. Association between rs2303861 polymorphism in CD82 gene and nonalcoholic fatty liver disease: a preliminary case-control study. Croat Med J 2019;60:361-8.

23. Baciu C, Pasini E, Angeli M, et al. Systematic integrative analysis of gene expression identifies HNF4A as the central gene in pathogenesis of non-alcoholic steatohepatitis. PLoS One 2017;12:e0189223.

24. Wu J, Zhou D, Deng C, et al. Characterization of porcine ENO3: genomic and cDNA structure, polymorphism and expression. Genet Sel Evol 2008;40:563-79.

25. Jia X, Zhai T. Integrated Analysis of Multiple Microarray
Studies to Identify Novel Gene Signatures in Nonalcoholic Fatty Liver Disease. Front Endocrinol (Lausanne) 2019;10:599.

26. Lu D, Yang Z, Xia Q, et al. ACADSB regulates ferroptosis and affects the migration, invasion, and proliferation of colorectal cancer cells. Cell Biol Int 2020;44:2334-43.

27. Kleiner DE, Brunt EM, Van Natta M, et al. Design and validation of a histological scoring system for nonalcoholic fatty liver disease. Hepatology 2005;41:1313-21.

28. Paul S, Davis AM. Diagnosis and Management of Nonalcoholic Fatty Liver Disease. JAMA 2018;320:2474-5.

29. Huang TD, Behary J, Zekry A. Non-alcoholic fatty liver disease: a review of epidemiology, risk factors, diagnosis and management. Intern Med J 2020;50:1038-47.

30. Hirsova P, Ibrahim SH, Gores GJ, et al. Lipotoxic lethal and sublethal stress signaling in hepatocytes: relevance to NASH pathogenesis. J Lipid Res 2016;57:1758-70. Erratum in: J Lipid Res 2017;58:299.

31. Başaranoğlu M, Ormeci N. Nonalcoholic fatty liver disease: diagnosis, pathogenesis, and management. Turk J Gastroenterol 2014;25:127-32.

32. Pár A, Par G. Advances in the pathogenesis of non alcoholic fatty liver disease. Orv Hetil 2017;158:882-94.

33. Ju L, Sun Y, Xue H, et al. CCN1 promotes hepatic steatosis and inflammation in non-alcoholic steatohepatitis. Sci Rep 2020;10:3201.

34. Helsley RN, Varadharajan V, Brown AL, et al. Obesitylinked suppression of membrane-bound O-acyltransferase 7 (MBOAT7) drives non-alcoholic fatty liver disease. Elife 2019;8:e49882.

35. Elam MB, Cowan GJ, Rooney RJ, et al. Hepatic gene expression in morbidly obese women: implications for disease susceptibility. Obesity (Silver Spring) 2009;17:1563-73.

36. Wei S, Qiu T, Wang N, et al. Ferroptosis mediated by the interaction between Mfn2 and IREalpha promotes arsenic-induced nonalcoholic steatohepatitis. Environ Res 2020;188:109824.

37. Conrad M, Kagan VE, Bayir H, et al. Regulation of lipid peroxidation and ferroptosis in diverse species. Genes Dev 2018;32:602-19.

38. Proneth B, Conrad M. Ferroptosis and necroinflammation, a yet poorly explored link. Cell Death Differ 2019;26:14-24.

39. Wu JR, Tuo QZ, Lei P. Ferroptosis, a Recent Defined Form of Critical Cell Death in Neurological Disorders. J Mol Neurosci 2018;66:197-206. 
40. Li D, Li Y. The interaction between ferroptosis and lipid metabolism in cancer. Signal Transduct Target Ther 2020;5:108.

41. Tesfay L, Paul BT, Konstorum A, et al. Stearoyl-CoA Desaturase 1 Protects Ovarian Cancer Cells from Ferroptotic Cell Death. Cancer Res 2019;79:5355-66.

42. Miess H, Dankworth B, Gouw AM, et al. The glutathione redox system is essential to prevent ferroptosis caused by impaired lipid metabolism in clear cell renal cell carcinoma. Oncogene 2018;37:5435-50.

43. Bai Y, Meng L, Han L, et al. Lipid storage and lipophagy regulates ferroptosis. Biochem Biophys Res Commun

Cite this article as: Lu D, Xia Q, Yang Z, Gao S, Sun S, Luo X, Li Z, Zhang X, Han S, Li X, Cao M. ENO3 promoted the progression of NASH by negatively regulating ferroptosis via elevation of GPX4 expression and lipid accumulation. Ann Transl Med 2021;9(8):661. doi: 10.21037/atm-21-471
2019;508:997-1003.

44. Lei P, Bai T, Sun Y. Mechanisms of Ferroptosis and Relations With Regulated Cell Death: A Review. Front Physiol 2019;10:139.

45. Ursini F, Maiorino M. Lipid peroxidation and ferroptosis: The role of GSH and GPx4. Free Radic Biol Med 2020;152:175-85.

46. Stockwell BR, Jiang X, Gu W. Emerging Mechanisms and Disease Relevance of Ferroptosis. Trends Cell Biol 2020;30:478-90.

(English Language Editor: A. Kassem) 